\title{
Fabry disease: a survey of visual and ocular symptoms
}

\section{Pinakin Gunvant Davey}

Western University of Health Sciences, College of Optometry, Pomona, CA, USA
Correspondence: Pinakin Gunvant Davey Western University of Health Sciences, College of Optometry, 309 E. Second Street, Pomona, CA, USA

Tel +I 9094698473

Email contact@pinakin-gunvant.com
This article was published in the following Dove Press journal:

Clinical Ophthalmology

19 August 2014

Number of times this article has been viewed

Purpose: To evaluate a visual symptoms survey on patients with a known diagnosis of Fabry disease, and to compare the scores to those from a group of healthy subjects.

Materials and methods: An ocular symptom survey instrument was used to evaluate the symptoms of general ocular problems like itching, tearing, dryness, burning sensation, sensation of foreign body, difficulty in scotopic and photopic vision, and asthenopic symptoms. The survey instrument was administered to 95 participants ( 75 patients with Fabry disease and 20 healthy controls; median age: 32.5 years [standard deviation: 19.1 years] and 42.6 years [standard deviation: 14.7 years], respectively). A Mann-Whitney test was performed to evaluate the difference between the Fabry group and the healthy controls for each symptom survey severity score. A $P$-value $<0.05$ was considered significant.

Results: Of the survey instrument items, it was found that compared to the control group, the mean severity score of the Fabry disease group was significant for "dryness" of the eyes $(P=0.02)$, "blurry/dim vision" $(P=0.02)$, "hard to see in dark places" $(P=0.01)$, and "halos around light" $(P=0.01)$. The Fabry group also had a mean severity score for "soreness/tiredness" that was significantly higher than that of the control group $(P=0.009)$.

Conclusion: The patients with Fabry disease may be suffering from ocular and visual symptoms related to manifestations of the disease. Further quantitative testing is needed to investigate the ocular and visual symptoms.

Keywords: Fabry disease, lysosomal storage disease, visual symptoms

\section{Introduction}

Fabry disease is a rare lysosomal disorder that has a prevalence rate affecting one in 40,000 males. ${ }^{1}$ This disease follows an X-linked inheritance pattern, and individuals affected with the disease (both males and females) show multisystem disorders that are present at birth, and the signs and symptoms worsen with time. ${ }^{1-4}$ The $G L A$ gene mutation leads to the deficiency of enzyme alpha galactidose $\mathrm{A}$, which leads to the progressive accumulation of globotriaosylceramide (GB3) deposits in a variety of cells including those in the kidneys, as well as in the autonomic and cardiovascular systems. ${ }^{2-4}$

The ocular manifestations of Fabry disease include cornea verticillata, which is the most common finding and is seen in all hemizygotes and in a majority (up to $70 \%$ ) of heterozygotes. ${ }^{5}$ Cornea verticillata is the outcome of deposits of GB3 in the basal layer of the corneal epithelium. ${ }^{5}$ Cornea verticillata is visible through a slit lamp, and an in vivo confocal microscope study has shown that even when cornea verticillata is not visible through a slit lamp, there are intracellular inclusion bodies in the basal epithelial cells. ${ }^{6}$ A smaller number of patients have subtle lenticular deposits, which are known as Fabry cataracts. ${ }^{7}$ Fabry cataracts tend to be off-axis dendritic or subcapsular opacities along the posterior suture lines. It is believed that patients with 
cornea verticillata will have no visual symptoms due to the corneal deposits. $^{7-9}$

The role of the cornea and crystalline lens is to provide the eye with necessary refractive power and it provides an unhindered path to the light rays that pass through them. The clear optical media, parallel and uniform arrangement of the collagen fibers ensure that the amount of scatter of light rays passing through the cornea is minimal. It can be hypothesized that individuals with Fabry disease could have problems with visual function given that both the cornea and crystalline lens have GB3 deposits and opacities. It can be further hypothesized that vision problems would be present and exaggerated at nighttime when compared to daytime vision, and they would occur with additional problems of glare, blurry vision, or dim vision (contrast sensitivity).

Patients with Fabry disease experience anhidrosis or hypohidrosis as one of the symptoms of the disease..$^{2-4}$ This is due to the neurological manifestations of the disease. Prior reports have suggested that patients with Fabry disease may have deposits of GB3 in the ganglia or the lacrimal gland itself. $^{10-12}$ It can be hypothesized that patients with Fabry disease could have symptoms of dry eyes.

An ocular symptom survey instrument was used to evaluate the symptoms associated with general ocular problems like itching, tearing, dryness, burning sensation, foreign body sensation, or difficulty in seeing in various conditions, as well as asthenopic symptoms, in patients with Fabry disease and healthy controls.

\section{Materials and methods Study participants}

A total of 95 individuals ( 75 patients with Fabry disease and 20 healthy controls) completed the survey. The study included 39 males ( 30 with the disease and nine controls) and 56 females (45 with the disease and eleven controls). The difference in the number of males to females was not statistically significant between groups (chi-square test, Pearson's $\chi^{2}$ statistic $=0.16$; $P=0.68$ ). The median age and standard deviation (SD) of the patients in the Fabry group and those in the control group were 32.5 years $(\mathrm{SD}=19.1$ years) and 42.6 years $(\mathrm{SD}=14.7$ years), respectively. Ninety-six percent of the participants completed all of the survey questions, with four individuals not answering the question about "soreness and fatigue" of the eyes.

The survey was administered live at the conferences for lysosomal disease storage, which were conducted by the Fabry Support and Information Group in San Diego, CA, USA, and by the National Fabry Disease Foundation in Greensboro, NC, USA. These events were attended by individuals that had a confirmed case of Fabry disease, or by a family member or spouse who had been tested and confirmed to be healthy and without Fabry disease. The participants were instructed to answer the survey questions with regard to their self-perceived ocular and vision status when they were wearing optimal refractive correction. Data were collated and analyzed in a masked fashion, with the data entry performed by a separate individual and the statistical analysis performed by the author.

\section{Survey instrument}

A modified survey instrument that was created by Lee et al ${ }^{13}$ to investigate the ocular and visual symptoms in glaucoma patients was utilized in the present study. The survey instrument proposed by Lee et $\mathrm{al}^{13}$ is called the Glaucoma Symptom Scale (GSS) and is a simple ten-question survey that was developed by modifying the survey used in the Ocular Hypertension Treatment Study. ${ }^{14}$ The GSS was tested and validated on a group of glaucoma patients in four tertiary care glaucoma centers. ${ }^{13}$

The survey administered in the present study is shown in Figure 1. This survey instrument was administered in the present study to both patients with Fabry disease and their healthy family members (controls). The major difference between the GSS and the survey administered in the present study is the scale of the symptoms. The GSS graded the symptoms on a four-point scale, whereas the present study used a six-point scale. The larger scale in the present survey allowed for the evaluation of a wide range of symptom levels, as it was likely that the symptoms were mild or minimal in patients with Fabry disease. The GSS survey instrument mainly evaluated dryness, as well as tear film and ocular surface issues in questions $1-4$ and 7 , whereas question 5 was designed to evaluate general fatigue or asthenopic symptoms. Questions 6 and 8-10 of the survey instrument were designed to evaluate vision-related problems - primarily, difficulty in contrast sensitivity, daytime/nighttime vision difficulties, and glare, respectively.

\section{Statistical analysis}

The difference in the mean age between the groups was analyzed using a one-way analysis of variance (ANOVA). The severity for each variable was graded on an ordinal scale, with 1 being "almost never" and 6 as "almost always". A Mann-Whitney test was performed to evaluate the median difference between those in the Fabry group and the healthy controls for each symptom survey question. A $P$-value $<0.05$ was considered significant. The statistical analysis was 
Almost never Almost always

\begin{tabular}{|l|l|l|l|l|l|l|l|}
\hline 1 & Burning/stinging & 1 & 2 & 3 & 4 & 5 & 6 \\
\hline 2 & Tearing & 1 & 2 & 3 & 4 & 5 & 6 \\
\hline 3 & Dryness & 1 & 2 & 3 & 4 & 5 & 6 \\
\hline 4 & Itching & 1 & 2 & 3 & 4 & 5 & 6 \\
\hline 5 & Soreness/tiredness & 1 & 2 & 3 & 4 & 5 & 6 \\
\hline 6 & Blurry/dim vision & 1 & 2 & 3 & 4 & 5 & 6 \\
\hline 7 & Feeling of something in your eyes & 1 & 2 & 3 & 4 & 5 & 6 \\
\hline 8 & Hard to see in daylight; I need to wear & 1 & 2 & 3 & 4 & 5 & 6 \\
\hline 9 & Hard to see in dark places & 1 & 2 & 3 & 4 & 5 & 6 \\
\hline 10 & Halos around light & 1 & 2 & 3 & 4 & 5 & 6 \\
\hline
\end{tabular}

Figure I Survey instrument administered to the study participants.

Notes: The participants read each numbered point and were instructed to grade the level of their symptom that is circle $I$ if they almost never had the problem and 6 is they always had a problem. Survey instrument developed by Lee et al adapted for this study. ${ }^{13}$

performed using Analyse-it ${ }^{\circledR}$ for Excel version 2.26 (Analyseit Software, Ltd., Leeds, UK).

\section{Results}

The mean age of the control group was greater than the mean age of the Fabry group (ANOVA $F=4.75 ; P=0.03$ ). Table 1 provides the mean symptom severity score for the Fabry group and the control group, as well as the $P$-values obtained using the Mann-Whitney test.

Of the survey instrument items, it was found that patients in the Fabry group reported significantly more "dryness" of the eyes compared to the control group $(P=0.02)$. The Fabry group also showed a significantly greater symptom severity score for "blurry/dim vision", "hard to see in dark places", and "halos around light" $(P=0.02 ; P=0.01$; and $P=0.01$, respectively). The Fabry group also had a mean severity score for "soreness/tiredness" that was significantly higher than that of the control group $(P=0.009)$. The tally of the number of participants with symptoms and without symptoms is given in Table $\mathrm{S} 1$.

\section{Discussion}

The present study utilized the GSS survey instrument developed by Lee et a ${ }^{13}$ to identify ocular and visual symptoms in Fabry disease and in healthy controls. The results of the present study indicate that compared to healthy controls, patients with Fabry disease had a greater ocular symptom severity score, especially in areas related to night vision, contrast sensitivity, and glare. Further, they also had a greater symptom severity score and complaints of soreness/tiredness and dry eyes.

Prior reports have indicated that patients with Fabry disease do not have any visual problems due to cornea verticillata. $^{7-9}$ This is perhaps true to the point that the presence of cornea verticillata may not cause any decline of

Table I Symptom surveys, the median severity of groups, and statistical significance

\begin{tabular}{lllll}
\hline No & Symptom & Mean severity score & \multicolumn{1}{c}{$\boldsymbol{P}$-value } \\
\cline { 3 - 5 } & & Fabry group & Control group \\
\hline 1 & Burning/stinging & 1.9 & 1.5 & 0.06 \\
2 & Tearing & 2.0 & 1.9 & 0.2 \\
3 & Dryness & 2.3 & 1.5 & $0.02 *$ \\
4 & Itching & 2.0 & 1.8 & 0.3 \\
5 & Soreness/tiredness & 2.4 & 1.7 & $0.009 * *$ \\
6 & Blurry/dim vision & 2.2 & 1.6 & $0.02 *$ \\
7 & Feeling of something in your eyes & 2.0 & 1.5 & 0.07 \\
8 & Hard to see in daylight; I need to wear sunglasses & 2.4 & 1.7 & 0.15 \\
9 & Hard to see in dark places & 2.6 & 1.6 & $0.0 I^{*}$ \\
10 & Halos around light & 2.4 & 1.7 & $0.0 I^{*}$ \\
\hline
\end{tabular}

Notes: *Significant at $P<0.05$; **ignificant at $P<0.01$. 
vision when measured using Snellen visual acuity charts, ${ }^{7-9}$ which use $100 \%$ contrast optotypes (black letters on a white background), as long as the patients wear appropriate correction for their refractive errors. The findings of this study indicate that the symptoms of "dim vision" and "hard to see in dark places" perhaps suggest that there is a decrease in contrast sensitivity or in identifying targets in day-to-day life that are in shades of grey and that are not made with $100 \%$ contrast. The Fabry group also had a symptom severity score that was significantly higher for the survey item addressing seeing "halo around lights", which is perhaps a result of the scatter or glare problems that the patients experience. This problem may perhaps be further exaggerated at nighttime.

The vision problems related to nighttime vision, contrast sensitivity, and glare are most likely due to the deposits of GB3 in the corneal epithelium and lens, which cause increased scatter of light rays and decreased transmittance of light (see Figures 2A-C). Confocal microscope study has revealed that even when there is no clinically visible cornea verticillata, the basal layer of the epithelium shows hyperreflectivity due to intracellular inclusion bodies. ${ }^{6}$ Thus, one can expect the symptom severity score to be related to the amount of corneal deposits that are visible; patients that do not have visible deposits may still have some visual problems given that they have intracellular inclusion bodies.

The Fabry group also had greater complaints of "dryness" when compared to the control group. This could be due to the fact that GB3 gets deposited both in the ganglia and in the lacrimal gland. ${ }^{10-12}$ Prior researchers have also suggested that patients with Fabry disease can have dry eye syndrome. ${ }^{9}$ The results of this study quantifies that patients may have a subtle, yet clinically significant, dry eye.

The patients with Fabry disease also complain of a lack of energy or chronic fatigue. ${ }^{15}$ It is intriguing to note that the symptom survey score for "soreness/tiredness of eyes" was significantly greater than in the control group. This could be due to multiple reasons. The simplest explanation lies in the fact that Fabry disease patients experience chronic fatigue of their body, and thus also experience "soreness/tiredness" with their eyes, although it is possible that dry eye and dim vision may contribute to some of these asthenopic problems. ${ }^{16}$ It will be interesting to see if this symptom severity score would decrease with appropriate therapy using glare filters, contrast-enhancing filters, or treatment for dry eyes.

Cornea verticillata in patients with Fabry disease can vary significantly; they can be absent, subtle, or dramatic in appearance. ${ }^{9}$ Figures $2 \mathrm{~A}-\mathrm{C}$ provide slit lamp photographs of cornea verticillata in patients with known Fabry disease.
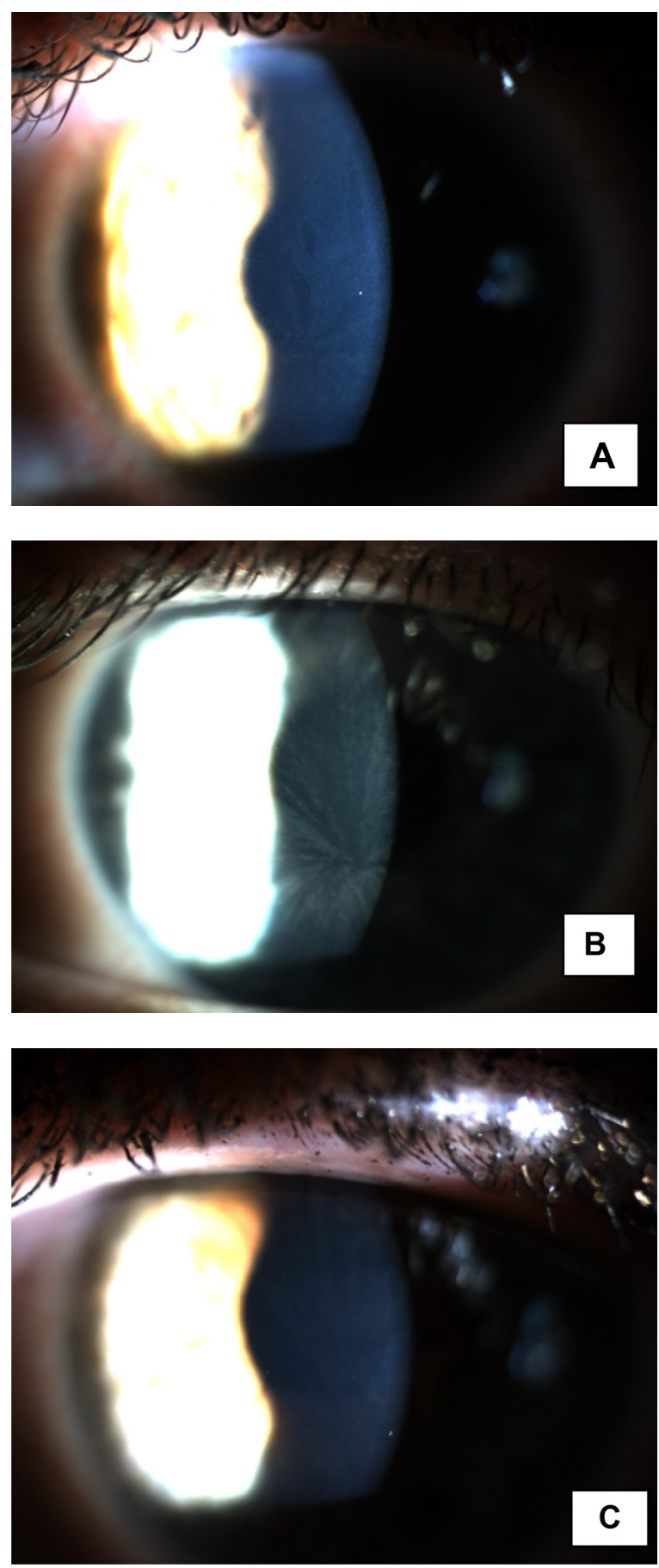

Figure 2 Cornea verticillata in patients with Fabry disease.

Notes: (A) Cornea verticillata giving an even spread of a smear of deposits throughout the cornea. (B) Vortex-like deposits. (C) A subtle verticillata in a patient with Fabry disease.

Figures $3 \mathrm{~A}$ and $\mathrm{B}$ are images of examples of conjunctival vascular findings in patients with Fabry disease. The conjunctival vascular findings are usually a late-onset finding and are seen in older patients with Fabry disease, rather than in younger patients. ${ }^{9}$ It should be noted that cornea verticillata is not limited to Fabry disease, and patients using medication like amiodarone or aminoquinolones on a chronic basis can have a similar corneal appearance. 7 It can be postulated that 

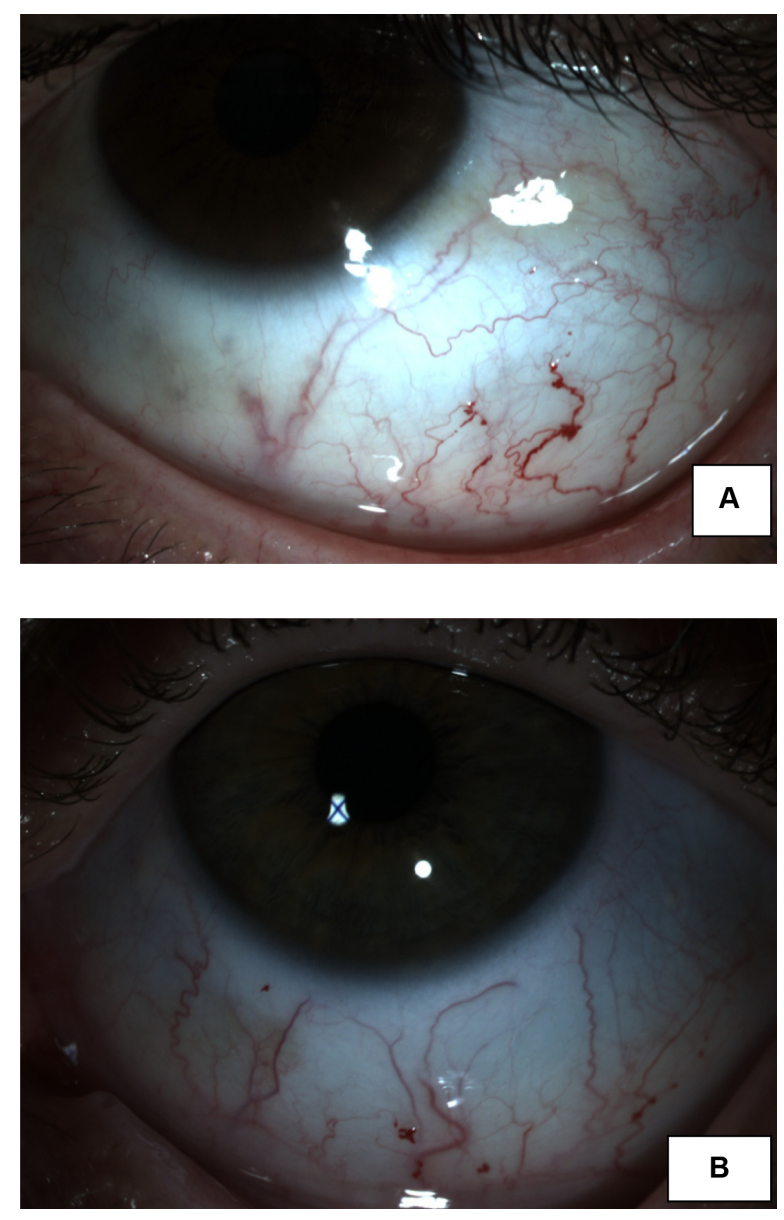

Figure 3 Conjunctival tortuosity with aneurysms presenting as an out-pouching. Notes: (A) Right eye of a patient with Fabry disease. The infero nasal bulbar conjunctiva shows conjunctival vessel tortuosity and aneurysm like outpouching. (B) Left eye of a patient with Fabry disease. The inferiorl bulbar conjunctiva shows conjunctival vessel tortuosity and aneurysm-like outpouching.

any person with cornea verticillata can have vision problems associated with nighttime vision, contrast discrimination, and glare.

This study was designed to evaluate whether ocular and visual symptoms were greater in patients with Fabry disease when compared to healthy controls. It was evident that the mean ocular symptom severity score was greater in the group with Fabry disease than in the control group. It will be interesting to research whether this is related to the type of mutation or the subset of cases (like classic Fabry, lateonset Fabry, or the variants). Further, it will be interesting to investigate if the ocular severity score is related to the severity of the disease, or if it varies as a function of the treatment administered. These questions remain to be answered and can possibly be addressed by future work in this area.

This is the first report, to my knowledge, that has evaluated ocular symptom severity in patients with Fabry disease. Future studies should look at quantifying the contrast sensitivity function and glare testing, and they should investigate tear function tests to evaluate vision-related difficulties and dry eye problems in patients with Fabry disease.

\section{Acknowledgments}

I would like to thank Mr Jerry Walter, Founder and President of the National Fabry Disease Foundation (NFDF), and Mr Jack Johnson, Executive Director of the Fabry Support and Information Group, for their kind help in administering the survey to Fabry patients. I would also like to thank Mr Nate Klingensmith of Haag Streit USA for his assistance in the ocular imaging of the Fabry group, Mr Jack Greenan for the data entry related to the study, and Dr Rebecca Kammer for her interesting discussions about survey instruments and disease.

\section{Disclosure}

The author reports no conflicts of interest in this work.

\section{References}

1. Meikle PJ, Hopwood JJ, Clague AE, Carey WF. Prevalence of lysosomal storage disorders. JAMA. 1999;281(3):249-254.

2. MacDermot KD, Holmes A, Miners AH. Anderson-Fabry disease: clinical manifestations and impact of disease in a cohort of 98 hemizygous males. J Med Genet. 2001;38(11):750-760.

3. MacDermot KD, Holmes A, Miners AH. Anderson-Fabry disease: clinical manifestations and impact of disease in a cohort of 60 obligate carrier females. J Med Genet. 2001;38(11):769-775.

4. Branton MH, Schiffmann R, Sabnis SG, et al. Natural history of Fabry renal disease: influence of alpha-galactosidase A activity and genetic mutations on clinical course. Medicine. 2002;81(2):122-138.

5. Desnick RJ, Brady RO. Fabry disease in childhood. J Pediatr. 2004; 144 (Suppl 5):S20-S26.

6. Wasielica-Poslednik J, Pfeiffer N, Reinke J, Pitz S. Confocal laserscanning microscopy allows differentiation between Fabry disease and amiodarone-induced keratopathy. Graefes Arch Clin Exp Ophthalmol. 2011;249(11):1689-1696.

7. Samiy N. Ocular features of Fabry disease: diagnosis of a treatable life-threatening disorder. Surv Ophthalmol. 2008;53(4):416-423.

8. Sodi A, Ioannidis A, Pitz S. Ophthalmological manifestations of Fabry disease. In: Mehta A, Beck M, Sunder-Plassmann G, editors. Fabry Disease: Perspectives From 5 Years of FOS. Oxford, UK: Oxford Pharmagenesis Ltd; 2006:249-261.

9. Sivley MD. Fabry Disease: a review of ophthalmic and systemic manifestations. Optom Vis Sci. 2013;90(2):e63-e78.

10. Cable WJ, Kolodny EH, Adams RD. Fabry disease: impaired autonomic function. Neurology. 1982;32(5):498-502.

11. Hilz MJ. Evaluation of peripheral and autonomic nerve function in Fabry disease. Acta Paediatr Suppl. 2002;91(439):38-42.

12. Kolodny EH, Pastores GM. Anderson-Fabry disease: extrarenal, neurologic manifestations. J Am Soc Nephrol. 2002;13(Suppl 2): S150-S153.

13. Lee BL, Gutierrez P, Gordon M, et al. The Glaucoma Symptom Scale A brief index of glaucoma-specific symptoms. Arch Ophthalmol. 1998; 116(7):861-866

14. Gordon MO, Beiser JA, Brandt JD, et al. The Ocular Hypertension Treatment Study: baseline factors that predict the onset of primary open-angle glaucoma. Arch Ophthalmol. 2002;120(6):714-720; discussion 829-830.

15. Zarate YA, Hopkin RJ. Fabry's disease. Lancet. 2008;372(9647): $1427-1435$.

16. McGinnigle S, Naroo SA, Eperjesi F. Evaluation of dry eye. Surv Ophthalmol. 2012;57(4):293-316. 


\section{Supplementary material}

Table SI A tally of the study participants that reported experiencing symptoms in the Fabry group and the control group

\begin{tabular}{|c|c|c|c|}
\hline Symptoms & & Problem & No problem \\
\hline \multirow[t]{2}{*}{ Burning and stinging } & Disease & 38 & 37 \\
\hline & No disease & 5 & 15 \\
\hline \multirow[t]{2}{*}{ Tearing } & Disease & 44 & 31 \\
\hline & No disease & 11 & 9 \\
\hline \multirow[t]{2}{*}{ Dryness* } & Disease & 44 & 31 \\
\hline & No disease & 7 & 13 \\
\hline \multirow[t]{2}{*}{ Itching } & Disease & 44 & 31 \\
\hline & No disease & 9 & II \\
\hline \multirow[t]{2}{*}{ Soreness, tiredness* } & Disease & 52 & 23 \\
\hline & No disease & 6 & 14 \\
\hline \multirow[t]{2}{*}{ Blurry, dim vision* } & Disease & 48 & 27 \\
\hline & No disease & 7 & 13 \\
\hline \multirow[t]{2}{*}{ Feeling something in your eyes } & Disease & 40 & 35 \\
\hline & No disease & 6 & 14 \\
\hline \multirow[t]{2}{*}{ Hard to see in daylight; I need sunglasses } & Disease & 39 & 36 \\
\hline & No disease & 7 & 13 \\
\hline \multirow[t]{2}{*}{ Hard to see in dark places* } & Disease & 45 & 30 \\
\hline & No disease & 7 & 13 \\
\hline \multirow[t]{2}{*}{ Halos around light* } & Disease & 44 & 31 \\
\hline & No disease & 6 & 14 \\
\hline
\end{tabular}

Notes: *Symptoms that had a statistically significant greater severity score between groups. The survey was administered to 95 individuals $(75$ Fabry disease and 20 healthy controls).

\section{Publish your work in this journal}

Clinical Ophthalmology is an international, peer-reviewed journal covering all subspecialties within ophthalmology. Key topics include: Optometry; Visual science; Pharmacology and drug therapy in eye diseases; Basic Sciences; Primary and Secondary eye care; Patient Safety and Quality of Care Improvements. This journal is indexed on
PubMed Central and CAS, and is the official journal of The Society of Clinical Ophthalmology (SCO). The manuscript management system is completely online and includes a very quick and fair peer-review system, which is all easy to use. Visit http://www.dovepress.com/ testimonials.php to read real quotes from published authors. 